\title{
A Domain Decomposition Method for Computing Bivariate Spline Fits of Scattered Data
}

\author{
Ming-Jun Lai ${ }^{1)}$ and Larry L. Schumaker ${ }^{2)}$
}

\begin{abstract}
A domain decomposition method for solving large bivariate scattered data fitting problems with bivariate minimal energy, discrete least-squares, and penalized least-squares splines is described. The method is based on splitting the domain into smaller domains, solving the associated smaller fitting problems, and combining the coefficients to get a global fit. Explicit error bounds are established for how well our locally constructed spline fits approximate the global fits. Some numerical examples are given to illustrate the effectiveness of the method.
\end{abstract}

\section{$\S 1$. Introduction}

Suppose $f$ is a smooth function defined on a domain $\Omega$ in $\mathbb{R}^{2}$ with polygonal boundary. Given the values $\left\{f_{i}:=f\left(x_{i}, y_{i}\right)\right\}_{i=1}^{n_{d}}$ of $f$ at some set of scattered points in $\Omega$, we consider the problem of computing a function $s$ that interpolates the data, or in the case of noisy data or large sets of data, approximates rather than interpolates $f$. There are many methods for solving this problem, but here we will focus on three methods based on bivariate splines, namely,

- the minimal energy (ME) method,

- the discrete least-squares (DLS) method,

- the penalized least-squares (PLS) method.

These three variational methods have been extensively studied in the literature, see $[1,6-8,12]$, and references therein. It is well known that all three do a good job of fitting smooth functions. But they are global methods, which means that the coefficients of a fitting spline are computed from a single linear system of equations which can be very large if the dimension of the spline space is large. This would appear to limit the applicability of variational spline methods to moderately sized problems. However, as we shall show in this paper, it is possible to

1) Department of Mathematics, The University of Georgia, Athens, GA 30602, mjlai@math.uga.edu. Partially supported by the National Science Foundation under Grant No. 0713807.

2) Department of Mathematics, Vanderbilt University, Nashville, TN 37240, larry.schumaker@vanderbilt.edu. 
efficiently compute ME-, DLS-, and PLS-splines, even with spline spaces of very large dimension.

Suppose that $\triangle$ is a triangulation of $\Omega$, and that $\mathcal{S}(\triangle)$ is a spline space defined on $\triangle$. Throughout this paper we assume that $\mathcal{S}(\triangle)$ has a stable local minimal determining set $\mathcal{M}$, see Sect. 4 or the book [10]. This means that each spline $s \in \mathcal{S}(\triangle)$ is uniquely determined by a set of coefficients $\left\{c_{\xi}\right\}_{\xi \in \mathcal{M}}$, where each $c_{\xi}$ is associated with a unique (domain) point $\xi$ of $\triangle$.

The idea of our method is simple. Instead of finding all of the coefficients $\left\{c_{\xi}\right\}_{\xi \in \mathcal{M}}$ at once, this algorithm reduces the problem to a collection of smaller problems. To state our algorithm formally, we need some additional notation. If $\omega$ is a subset of $\Omega$, we set $\operatorname{star}^{0}(\omega)=\bar{\omega}$, and recursively define

$$
\operatorname{star}^{\ell}(\omega):=\bigcup\left\{T \in \triangle: T \cap \operatorname{star}^{\ell-1}(\omega) \neq \emptyset\right\}
$$

for all $\ell \geq 1$.

\section{Algorithm 1.1. (Domain Decomposition Method)}

1) Choose a decomposition of $\Omega$ into disjoint connected sets $\left\{\Omega_{i}\right\}_{i=1}^{m}$.

2) Choose $k>0$. For each $i=1, \ldots, m$, let $\left.s_{i}^{k} \in \mathcal{S}(\triangle)\right|_{\Omega_{i}^{k}}$ be the spline fit based on data in $\Omega_{i}^{k}:=\operatorname{star}^{k}\left(\Omega_{i}\right)$. Let $\left\{c_{i, \xi}^{k}\right\}$ be the set of all coefficients of $s_{i}^{k}$.

3) For each $i=1, \ldots, m$, set

$$
c_{\xi}=c_{i, \xi}^{k}, \quad \text { all } \xi \in \mathcal{M} \cap \Omega_{i}
$$

We call a spline $s$ produced by this algorithm a domain decomposition (DDC) spline. We emphasize that this domain decomposition method is very different from domain decomposition methods used in classical numerical methods for partial differential equations and in the application of radial basis functions to scattered data fitting and meshless methods for PDE's, see Remark 1. As we shall see, our method

- is easy to implement,

- allows the solution of very large data fitting problems,

- with appropriately chosen $m$ and $k$, produces a spline which is very close to the globally defined spline,

- is amenable to parallel processing,

- produces a spline $s$ in the space $\mathcal{S}(\triangle)$, i.e., with the same smoothness as the global fit,

- does not make use of blending functions.

The paper is organized as follows. In Sect. 2 we review the basics of minimal energy, discrete least-squares, and penalized least-squares spline fitting. Then in Sect. 3 we present some numerical experiments to illustrate the performance of our domain decomposition method. There we also explore the following questions: 
- how does the time required to compute a domain-decomposition spline $s$ compare with that required for finding a global spline fit $s_{g}$ from $\mathcal{S}(\triangle)$ ?

- how does $\left\|s-s_{g}\right\|$ behave as we choose different decompositions and different values for the parameter $k$ ?

- how well does the shape of $s$ match that of $s_{g}$ ?

In Sect. 4 we review some Bernstein-Bézier tools needed to analyze our method and present two lemmas needed later. In Sect. 5 we show that for the variational spline methods described in the following section, $\left\|s-s_{g}\right\|=\mathcal{O}\left(\sigma^{k}\right)$ for some $0<\sigma<1$. We conclude the paper with remarks and references.

\section{$\S 2$. Three Variational Spline Fitting Methods}

Given $d>r \geq 1$ and a triangulation $\triangle$ of a domain $\Omega \in \mathbb{R}^{2}$, let

$$
\mathcal{S}_{d}^{r}(\triangle):=\left\{s \in C^{r}(\Omega):\left.s\right|_{T} \in \mathcal{P}_{d}, \text { all } T \in \triangle\right\}
$$

be the associated space of bivariate splines of smoothness $r$ and degree $d$. Here $\mathcal{P}_{d}$

is the $\left(\begin{array}{c}d+2 \\ 2\end{array}\right)$ dimensional space of bivariate polynomials of degree $d$. Such spaces, along with various subspaces of so-called supersplines, have been intensely studied in the literature, see the book [10] and references therein. There are many splinebased methods for interpolation and approximation. Here we are interested in three particular methods.

\subsection{Minimal energy interpolating splines}

Suppose we are given values $\left\{f_{i}\right\}_{i=1}^{n_{d}}$ associated with a set of $n_{d} \geq 3$ abscissae $\mathcal{A}:=\left\{\left(x_{i}, y_{i}\right)\right\}_{i=1}^{n_{d}}$ in the plane. The problem is to construct a smooth function $s$ that interpolates this data in the sense that

$$
s\left(x_{i}, y_{i}\right)=f_{i}, \quad i=1, \ldots, n_{d} .
$$

To solve this problem, suppose $\triangle$ is a triangulation with vertices at the points of $\mathcal{A}$. Let $\mathcal{S}(\triangle)$ be a spline space defined on $\triangle$ with dimension $n \geq n_{d}$, and let

$$
\Lambda(f)=\left\{s \in \mathcal{S}(\triangle): s\left(x_{i}, y_{i}\right)=f_{i}, i=1, \cdots, n_{d}\right\}
$$

Let

$$
E(s)=\int_{\Omega}\left[\left(s_{x x}\right)^{2}+2\left(s_{x y}\right)^{2}+\left(s_{y y}\right)^{2}\right] d x d y
$$

be the well-known thin-plate energy of $s$. Then the minimal energy (ME) interpolating spline is the function $s_{E}$ in $\Lambda$ such that

$$
E\left(s_{E}\right)=\min _{s \in \Lambda(f)} E(s)
$$


Assuming $\Lambda(f)$ is nonempty, it is well known (see e.g. [1,7,12]) that there exists a unique ME-spline characterized by the property

$$
\left\langle s_{E}, g\right\rangle_{E}=0, \quad \text { all } g \in \Lambda(0),
$$

where

$$
\langle\phi, \psi\rangle_{E}=\int_{\Omega}\left[\phi_{x x} \psi_{x x}+2 \phi_{x y} \psi_{x y}+\phi_{y y} \psi_{y y}\right] d x d y .
$$

Moreover, its Bernstein-Bézier coefficients can be computed by solving an appropriate linear system of equations. For details on two different approaches to this computation, see [1] and [12].

Assuming the data come from a smooth function, i.e.,

$$
f_{i}=f\left(x_{i}, y_{i}\right), \quad i=1, \ldots, n_{d},
$$

it is possible to give an error bound for how well the corresponding minimal energy interpolating spline $s_{e}$ approximates $f$. To state the result, suppose the triangulation $\triangle$ is $\beta$-uniform, i.e.,

$$
\frac{|\triangle|}{\rho_{\triangle}} \leq \beta<\infty,
$$

where $|\triangle|$ is the length of the longest edge in $\triangle$, and $\rho_{\triangle}$ is the minimum of the inradii of the triangles of $\triangle$. Let $\theta_{\triangle}$ be the smallest angle in $\triangle$. Then it was shown in Theorem 6.2 of [7] that for all $f \in W_{\infty}^{2}(\Omega)$,

$$
\left\|f-s_{E}\right\|_{\Omega} \leq C|\triangle|^{2}|f|_{2, \Omega},
$$

where $\|\cdot\|_{\Omega}$ is the supremum norm on $\Omega$, and $|\cdot|_{2, \Omega}$ is the corresponding Sobolev semi-norm. $C$ is a constant depending only on $d, \ell, \beta$ and $\theta_{\triangle}$ if $\Omega$ is convex. If $\Omega$ is nonconvex, the constant $C$ may also depend on the Lipschitz constant of the boundary of $\Omega$.

Now suppose $s_{E}^{k}$ is a DDC minimal energy spline computed using Algorithm 1.1 with parameter $k \geq \ell$. Then since the analog of (2.7) holds for each subdomain $\Omega_{i}$ of $\Omega$, we have

$$
\left\|s_{E}-s_{E}^{k}\right\|_{\Omega} \leq C|\triangle|^{2}|f|_{2, \Omega} .
$$

This shows that the DDC minimal energy spline $s_{E}^{k}$ interpolating a given function $f$ is close to the global minimal energy spline $s_{E}$ whenever $f$ is smooth and $|\triangle|$ is small. The estimate (2.8) does not depend on $k$, and so gives no information on how the difference behaves with increasing $k$. In Sect. 5.1 we show that $\left\|s_{E}-s_{E}^{k}\right\|_{\Omega}=$ $\mathcal{O}\left(\sigma^{k}\right)$ with $0<\sigma<1$. 


\subsection{Discrete least-squares splines}

When the set of data is very large or the measurements $\left\{f_{i}\right\}_{i=1}^{n_{d}}$ are noisy, it is often better to construct an approximation from a spline space $\mathcal{S}(\triangle)$ of dimension $n<n_{d}$. Some or all of the vertices of $\triangle$ may be at points in $\mathcal{A}:=\left\{\left(x_{i}, y_{i}\right)\right\}_{i=1}^{n_{d}}$, but they may also be completely different. The solution of the variational problem of minimizing

$$
\|s-f\|_{\mathcal{A}}^{2}:=\sum_{j=1}^{n_{d}}\left[s\left(x_{j}, y_{j}\right)-f_{j}\right]^{2}
$$

over all $s$ in $\mathcal{S}(\triangle)$ is called the discrete least-squares spline $s_{L}$. It is well known, see e.g. $[1,12]$ that if $\mathcal{S}(\triangle)$ satisfies the property

$$
s\left(x_{i}, y_{i}\right)=0, \quad i=1, \ldots, n_{d}, \quad \text { implies } s \equiv 0,
$$

then there is a unique discrete least-squares spline $s_{L}$ fitting the data. It is characterized by the property

$$
\left\langle s_{L}-f, g\right\rangle_{\mathcal{A}}=0, \quad \text { all } g \in \mathcal{S}(\triangle),
$$

where

$$
\langle\phi, \psi\rangle_{\mathcal{A}}:=\sum_{i=1}^{n_{d}} \phi\left(x_{i}, y_{i}\right) \psi\left(x_{i}, y_{i}\right) .
$$

The Bernstein-Bézier coefficients of $s_{L}$ can be computed by solving an appropriate linear system of equations. For details on two different approaches to this computation, see [1] and [12].

Assuming the data come from a smooth function, it is possible to give an error bound for how well the least-squares spline $s_{L}$ approximates $f$. To state the result, suppose as before that the triangulation $\triangle$ is $\beta$-uniform. In addition, suppose that the data is sufficiently dense that for some constant $K_{1}>0$,

$$
K_{1}\|s\|_{T} \leq\left(\sum_{\left(x_{j}, y_{j}\right) \in T} s\left(x_{j}, y_{j}\right)^{2}\right)^{1 / 2}, \quad \text { for all } s \in \mathcal{S}(\triangle) \text { and all } T \in \triangle .
$$

Let

$$
K_{2}:=\max _{T \in \triangle} \#(\mathcal{A} \cap T) .
$$

Then for all $f \in W_{\infty}^{m+1}(\Omega)$ with $0 \leq m \leq d$,

$$
\left\|f-s_{L}\right\|_{\Omega} \leq C|\triangle|^{m+1}|f|_{m+1, \Omega},
$$

see the remark following Theorem 8.1 in [6]. If $\Omega$ is convex, the constant $C$ depends only on $d, \ell, \beta, K_{2} / K_{1}$ and $\theta_{\triangle}$. If $\Omega$ is nonconvex, $C$ may also depend on the Lipschitz constant of the boundary of $\Omega$. 
Now suppose $s_{L}^{k}$ is a DDC least-squares spline computed using Algorithm 1.1 with parameter $k \geq \ell$. Then the same error bound holds for each subdomain $\Omega_{i}$ of $\Omega$, and combining with (2.13) gives

$$
\left\|s_{L}-s_{L}^{k}\right\|_{\Omega} \leq C|\triangle|^{m+1}|f|_{m+1, \Omega} .
$$

This shows that the DDC least-squares spline $s_{L}^{k}$ fitting measurements of a given function $f$ is close to the global minimal energy spline $s_{L}$ whenever $f$ is smooth and $|\triangle|$ is small. The estimate (2.14) does not depend on $k$, and so gives no information on how the difference behaves with increasing $k$. In Sect. 5.2 we show that it is $\mathcal{O}\left(\sigma^{k}\right)$ with $0<\sigma<1$.

\subsection{Penalized least-squares splines}

Suppose $\mathcal{A}:=\left\{x_{i}, y_{i}\right\}_{i=1}^{n_{d}}$ and $\mathcal{S}(\triangle)$ are as in the previous subsections. Fix $\lambda \geq 0$. Then given data values $\left\{f_{i}\right\}_{i=1}^{n_{d}}$, the corresponding penalized least-squares (PLS) spline is defined to be the spline $s_{\lambda}$ in $\mathcal{S}(\triangle)$ that minimizes

$$
E_{\lambda}(s):=\|s-f\|_{\mathcal{A}}+\lambda E(s),
$$

where $E(s)$ is defined in (2.1). It is well known, cf. [1,12] that if $\mathcal{S}$ is a spline space such that $(2.9)$ holds, then there exists a unique PLS-spline $s_{\lambda}$ minimizing $E_{\lambda}(s)$ over $s \in \mathcal{S}(\triangle)$. Moreover, $s_{\lambda}$ is characterized by

$$
\left\langle s_{\lambda}-f, s\right\rangle_{\mathcal{A}}+\lambda\left\langle s_{\lambda}, s\right\rangle_{E}=0, \quad \text { all } s \in \mathcal{S}(\triangle) .
$$

As with the other two methods, the Bernstein-Bézier coefficients of $s_{\lambda}$ can be computed by solving an appropriate linear system of equations. For details on two different approaches to this computation, see [1] and [12]. It is known [8] that for all $f \in W_{\Omega}^{m+1}$ with $0 \leq m \leq d$,

$$
\left\|f-s_{\lambda}\right\|_{\Omega} \leq C\left(|\triangle|^{m+1}|f|_{m+1, \Omega}+\lambda|f|_{2, \Omega}\right) .
$$

for $\lambda$ sufficiently small compared to $|\triangle|$. The constant $C$ depends only on $d, \ell, \beta, \theta_{\triangle}$, $K_{2} / K_{2}$ and the area of $\Omega$. If $\Omega$ is nonconvex, $C$ may also depend on the Lipschitz constant of the boundary of $\Omega$.

Now suppose $s_{\lambda}^{k}$ is a DDC penalized least-squares spline computed using Algorithm 1.1 with parameter $k \geq \ell$. Then since the analog of (2.16) holds for each subdomain $\Omega_{i}$ of $\Omega$, we have

$$
\left\|s_{\lambda}-s_{\lambda}^{k}\right\|_{\Omega} \leq C\left(|\triangle|^{m+1}|f|_{m+1, \Omega}+\lambda|f|_{2, \Omega}\right) .
$$

This shows that the DDC penalized least-squares spline $s_{\lambda}^{k}$ fitting a given function $f$ is close to the global penalized least-squares spline $s_{\lambda}$ whenever $f$ is smooth and $|\triangle|$ is small. The estimate (2.17) does not depend on $k$, and so gives no information on how the difference behaves with increasing $k$. In Sect. 5.3 we show that it is $\mathcal{O}\left(\sigma^{k}\right)$ with $0<\sigma<1$. 


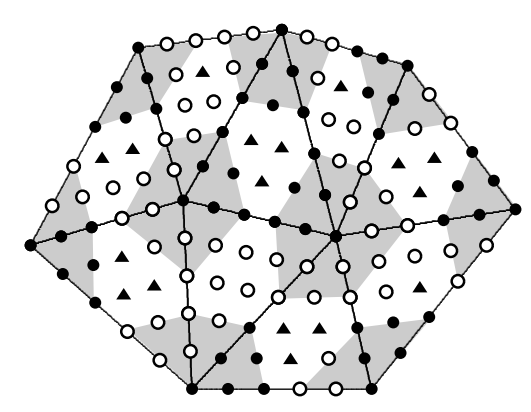

Fig. 1. A minimal determining set for $\mathcal{S}_{5}^{1,2}(\triangle)$.

\section{$\S 3$. Numerical Examples}

In this section we illustrate the domain decomposition method by applying it to compute minimal energy and discrete least-squares fits of scattered data. All of our examples are based on the superspline space

$$
\mathcal{S}_{5}^{1,2}(\triangle):=\left\{s \in \mathcal{S}_{5}^{1}(\triangle): s \in C^{2}(v) \text { for all vertices } v \in \triangle\right\}
$$

Here $s \in C^{2}(v)$ means that all polynomial pieces of $s$ on triangles sharing the vertex $v$ have common derivatives up to order 2 at $v$. It is well known that the dimension of this space is $6 n_{V}+n_{E}$, where $n_{V}, n_{E}$ are the number of vertices and edges of $\triangle$, respectively. The computations in this section are based on the algorithms in [12] which make use of a stable local minimal determining set $\mathcal{M}$ for $\mathcal{S}_{5}^{1,2}(\triangle)$ and the associated stable local $\mathcal{M}$-bases defined in [10]. Fig. 1 shows a minimal determining set for $\mathcal{S}_{5}^{1,2}(\triangle)$, where points in the set are marked with black dots and triangles.

\subsection{Example 1}

Let $H$ be the unit square, and let

$$
\begin{aligned}
F(x, y)= & 0.75 \exp \left(-0.25(9 x-2)^{2}-0.25(9 y-2)^{2}\right) \\
& +0.75 \exp \left(-(9 x+1)^{2} / 49-(9 y+1) / 10\right) \\
& +0.5 \exp \left(-0.25(9 x-7)^{2}-0.25(9 y-3)^{2}\right) \\
& -0.2 \exp \left(-(9 x-4)^{2}-(9 y-7)^{2}\right)
\end{aligned}
$$

be the well-known Franke function defined on $H$, see Fig. 2. Let $\triangle_{1087}$ be the triangulation shown in Fig. 3, This triangulation has 1087 vertices, 3130 edges, and 2044 triangles. The dimension of the space $\mathcal{S}_{5}^{1,2}\left(\triangle_{1087}\right)$ is 9652 , and the total number of Bernstein-Bézier coefficients of a spline in this space is 25,871 .

First we compute the minimal energy spline fit $s_{E}$ of $f$ from $\mathcal{S}_{5}^{1,2}\left(\triangle_{1087}\right)$. This requires solving a linear system of 8565 equations with 322,989 nonzero entries. Although the largest element in the corresponding matrix is $\mathcal{O}\left(10^{7}\right)$, its condition number is of order $\mathcal{O}\left(10^{4}\right)$. For comparison purposes we computed the maximum 


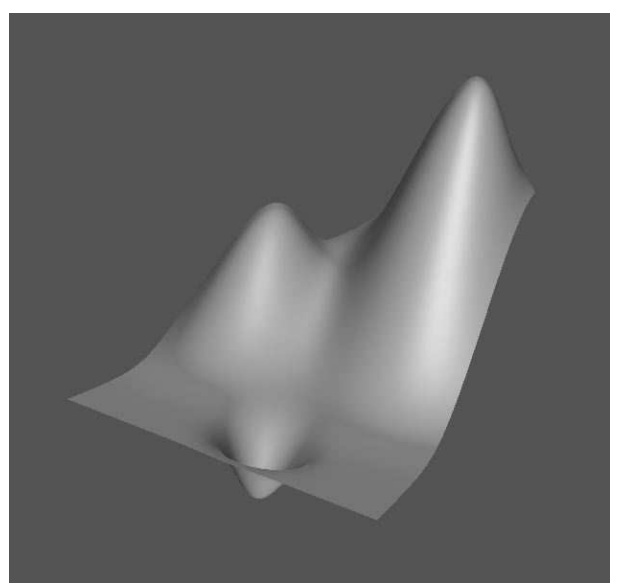

Fig. 2. The Franke function.

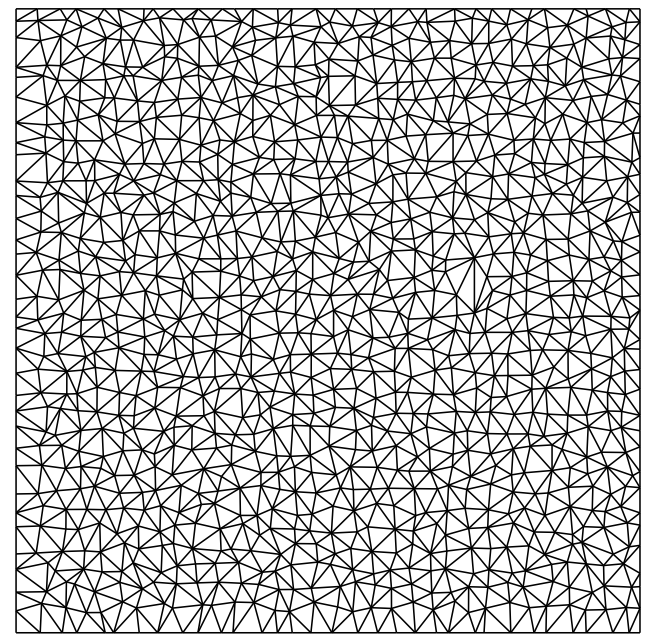

Fig. 3. A triangulation of 1087 vertices.

error $e_{\infty}$ over a $160 \times 160$ grid, along with the RMS error $e_{2}$ over the same grid. These errors are shown in the first line of Tab. 1, along with the computational time in seconds.

To explore the performance of our domain-decomposition technique, we computed approximations of $s_{E}$ by decomposing $\Omega$ into squares $\left\{\Omega_{i}\right\}_{i=1}^{m^{2}}$ of width $1 / \mathrm{m}$. In Tab. 1 we list the results, where $k$ is the parameter controlling the size of the sets $\Omega_{i}^{k}$ in Algorithm 1.1. In addition to the errors $e_{\infty}$ and $e_{2}$ measuring how well $s_{E}$ fits $f$, we also tabulate the maximum difference $e_{\infty}^{c}$ between the coefficients of our DDC spline and the coefficients of the global minimal energy spline $s_{E}$. We also compute the RMS difference $e_{2}^{c}$ for the coefficients, and list the computational time in seconds. We now comment on these results. 


\begin{tabular}{||c||c||c||c||c||c||c||}
\hline$m$ & $k$ & $e_{\infty}$ & $e_{2}$ & $e_{\infty}^{c}$ & $e_{2}^{c}$ & time \\
\hline 1 & 0 & $9.1(-4)$ & $7.7(-5)$ & & & 25 \\
4 & 1 & $3.0(-3)$ & $2.1(-4)$ & $8.5(-3)$ & $9.1(-5)$ & 9 \\
4 & 2 & $9.3(-4)$ & $8.6(-5)$ & $3.4(-3)$ & $1.9(-5)$ & 15 \\
4 & 3 & $9.1(-4)$ & $7.8(-5)$ & $3.4(-4)$ & $3.0(-6)$ & 21 \\
4 & 4 & $9.1(-4)$ & $7.7(-5)$ & $5.4(-5)$ & $4.4(-7)$ & 30 \\
8 & 1 & $3.1(-3)$ & $2.7(-4)$ & $8.6(-3)$ & $1.6(-4)$ & 7 \\
8 & 2 & $9.2(-4)$ & $9.4(-5)$ & $1.9(-3)$ & $3.5(-5)$ & 16 \\
8 & 3 & $9.1(-4)$ & $7.8(-5)$ & $3.4(-4)$ & $7.0(-6)$ & 29 \\
8 & 4 & $9.1(-4)$ & $7.7(-5)$ & $8.9(-5)$ & $1.3(-7)$ & 50 \\
\hline
\end{tabular}

Tab. 1. DDC minimal energy fits to Franke's function from $\mathcal{S}_{5}^{1,2}\left(\triangle_{1087}\right)$.

- Accuracy of fit: The table shows that in this experiment, the DDC splines with $k=1$ do not fit $f$ as well as the minimal energy spline, but as soon as $k \geq 2$, the errors are virtually identical. From the standpoint of accuracy of fit, there is no need to use values of $k$ larger than 2 or 3 .

- Accuracy of coefficients: The table shows that the DDC fits also provide very good approximations of the coefficients of the global minimal energy spline $s_{E}$. Both $e_{\infty}^{c}$ and $e_{2}^{c}$ decrease as $k$ increases, as predicted by the theoretical results in Sect. 5.1.

- Time: The main use of the DDC algorithm is to make it possible to solve large variational spline problems which could not be solved at all without using the method. For small problems, it often takes more time to solve for a DDC minimal energy spline than for the global minimal energy spline itself. For this moderately sized problem, we see that some of the DDC splines took less time to compute than the global fit, even for the same accuracy. For example, the DDC spline with $m=8$ and $k=2$ delivers virtually the same accuracy as the global minimal energy spline, but in only about one-half the computing time. For larger problems, the time required to compute DDC minimal energy splines is substantially less than for the global splines, see Example 2.

- Condition numbers: Since the entries in the matrix of the linear systems depend on integrals of squares of second derivatives over triangles, when the triangles are of size $\mathcal{O}(h)$, the entries are of size $\mathcal{O}\left(h^{-4}\right)$ and even larger if triangles are very thin. In this example the largest entries are on the order $\mathcal{O}\left(10^{7}\right)$. For very regular triangulations (for example type-I triangulations), the condition numbers of the matrices are of size $\mathcal{O}\left(10^{3}\right)$, independent of how many triangles there are. For less regular triangulations, they can be much larger. However, for the matrices associated with the triangulations in Fig. 4, they are of order $\mathcal{O}\left(10^{4}\right)$. 

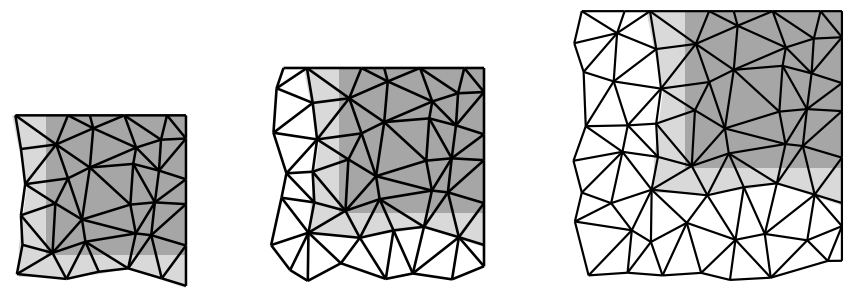

Fig. 4. $\operatorname{star}^{k}\left(\Omega_{64}\right)$ for $k=1,2,3$.

- Shape of $\operatorname{star}^{k}:$ Fig. 4 shows $\operatorname{star}^{k}\left(\Omega_{64}\right)$ for $k=1,2,3$, where $\Omega_{64}:=[.875,1] \times$ $[.875,1]$, shown in dark grey in the figure. The white triangles are the triangles added to form the stars.

- Shape of the surface: We have compared 3D plots of the global minimal energy fit of $f$ with the DDC-minimal energy fits for the parameters in Tab. 1. For $k=1$ we noticed slight deviations in shape, but for all higher values of $k$ we got excellent shapes.

\subsection{Example 2}

We repeat Example 1 with a type-I triangulation of the unit square with 4225 vertices. This triangulation includes 12,416 edges, and 8,192 triangles. The dimension of the space $\mathcal{S}_{5}^{1,2}\left(\triangle_{4225}\right)$ is 37,776 , and the total number of Bernstein-Bézier coefficients of a spline in this space is 103,041. We again fit the Franke function.

First we compute the minimal energy spline fit $s_{E}$ of $f$ from $\mathcal{S}^{1,2}\left(\triangle_{4225}\right)$. This requires solving a linear system of 33,541 equations with $1,282,073$ nonzero entries. Although the largest element in this matrix is $\mathcal{O}\left(10^{7}\right)$, its condition number is $\mathcal{O}\left(10^{4}\right)$. Our program took 326 seconds to compute $s$. For comparison purposes, we computed the maximum error $e_{\infty}$ over a $160 \times 160$ grid, along with the RMS error $e_{2}$ over this grid. These errors are shown in the first line of Tab. 2, along with the computational time (in seconds).

We computed approximations of $s_{E}$ using the same decompositions of $\Omega$ as in Example 1 based on $m^{2}$ squares of width $1 / m$. In Tab. 2 we list the results. Here we see that using the DDC method results in substantial time savings. We also see that the errors $e_{\infty}^{c}$ and $e_{2}^{c}$ behave like $\mathcal{O}\left(\sigma^{k}\right)$ with $\sigma \approx 1 / 4$, confirming the theoretical results in Sect. 5.2.

\subsection{Example 3}

In this example we work with elevation heights measured at 15,585 points in the Black Forest of Germany. The corresponding DeLaunay triangulation $\triangle_{B F}$ is shown in Fig. 5, although the triangulation is so fine in many areas that it is impossible to see the individual triangles without zooming in. This triangulation has 47,333 edges and 31,449 triangles. The dimension of the space $\mathcal{S}_{5}^{1,2}\left(\triangle_{B F}\right)$ is 142,643 , and the total number of Bernstein-Bézier coefficients of a spline in this space is 393,911 . 


\begin{tabular}{||c||c||c||c||c||c||c||}
\hline$m$ & $k$ & $e_{\infty}$ & $e_{2}$ & $e_{\infty}^{c}$ & $e_{2}^{c}$ & time \\
\hline 1 & 0 & $1.2(-4)$ & $7.6(-6)$ & & & 326 \\
8 & 1 & $9.9(-4)$ & $4.7(-5)$ & $2.2(-3)$ & $2.3(-5)$ & 37 \\
8 & 2 & $2.9(-4)$ & $1.5(-5)$ & $6.8(-4)$ & $5.7(-6)$ & 65 \\
8 & 3 & $1.8(-4)$ & $9.9(-6)$ & $1.7(-4)$ & $1.4(-6)$ & 97 \\
16 & 1 & $9.8(-4)$ & $6.9(-5)$ & $2.3(-3)$ & $4.4(-5)$ & 29 \\
16 & 2 & $2.9(-4)$ & $1.9(-5)$ & $7.6(-4)$ & $1.0(-5)$ & 66 \\
16 & 3 & $1.8(-4)$ & $1.0(-5)$ & $1.6(-4)$ & $2.5(-6)$ & 128 \\
\hline
\end{tabular}

Tab. 2. DDC minimal energy fits to Franke's function from $\mathcal{S}_{5}^{1,2}\left(\triangle_{4225}\right)$.

The computation of the minimal energy spline fit $s_{E}$ would require solving a linear system of 126,758 equations, which is beyond the capability of our software. So instead we computed a DDC approximation of the minimal energy spline using the decomposition of Example 1 based on 100 squares. The computation took 288 seconds, and Fig. 6 shows the resulting surface.

\subsection{Example 4}

In this example we again work on the unit square $H$. This time we approximate Franke's function by least squares based on measured data at 62,500 grid points in $H$. We approximate from the space $\mathcal{S}_{2}^{1}\left(\triangle_{1087}\right)$, where $\triangle_{1087}$ is the same triangulation as in Example 1, see Fig. 3. We choose this triangulation since it is big enough to illustrate how the domain decomposition method works, but small enough so that we can compute the global least square spline for comparison purposes. This function can of course be fit very well with much smaller spline spaces and much less data. For example with a type-I triangulation with 81 vertices and 1089 grid data, the errors for the least-squares spline fit are $e_{\infty}=5.2(-4)$ and $e_{2}=5.0(-5)$. The results of our experiments are shown in Tab. 3. Note that the times of computation for least-squares splines are significantly greater than for the minimal energy splines reported on in Tab. 1. This is due to the fact that a large part of the computation is taken up with finding the triangles containing the various data points. These times can be reduced by incorporating standard techniques for reducing the time required for these search operations.

- Accuracy of fit: The table shows that in this experiment the DDC least-squares splines with $k=1$ do not fit $f$ quite as well as the global least-squares spline, but with increasing $k$ they come very close. As with the minimal energy case, it appears that a good choice might be $k=2$.

- Accuracy of coefficients: The table shows that the DDC fits also provide very good approximations of the coefficients of the global least-squares spline. Both $e_{\infty}^{c}$ and $e_{2}^{c}$ decrease as $k$ increases. Indeed, for $m=4$, the error of $e_{\infty}^{c}$ behaves like $\mathcal{O}\left(\sigma^{k}\right)$ with $\sigma \approx 1 / 4$, while for $m=8, \sigma \approx 2$. There is a similar effect for $e_{2}$, confirming the theoretical results in Sect. 5.2. 


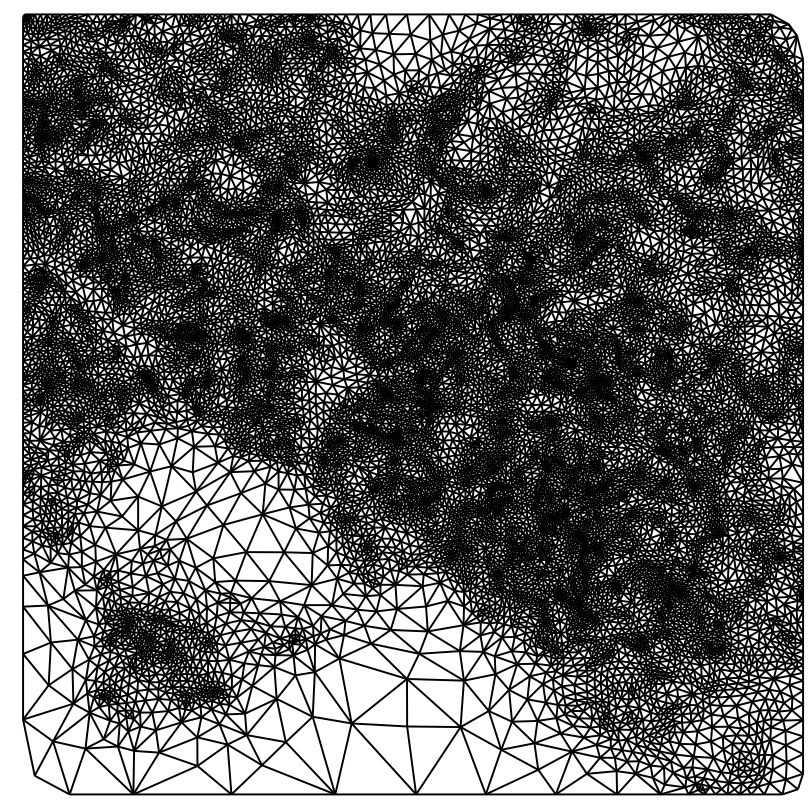

Fig. 5. Triangulation of 15585 points in the Black Forest.

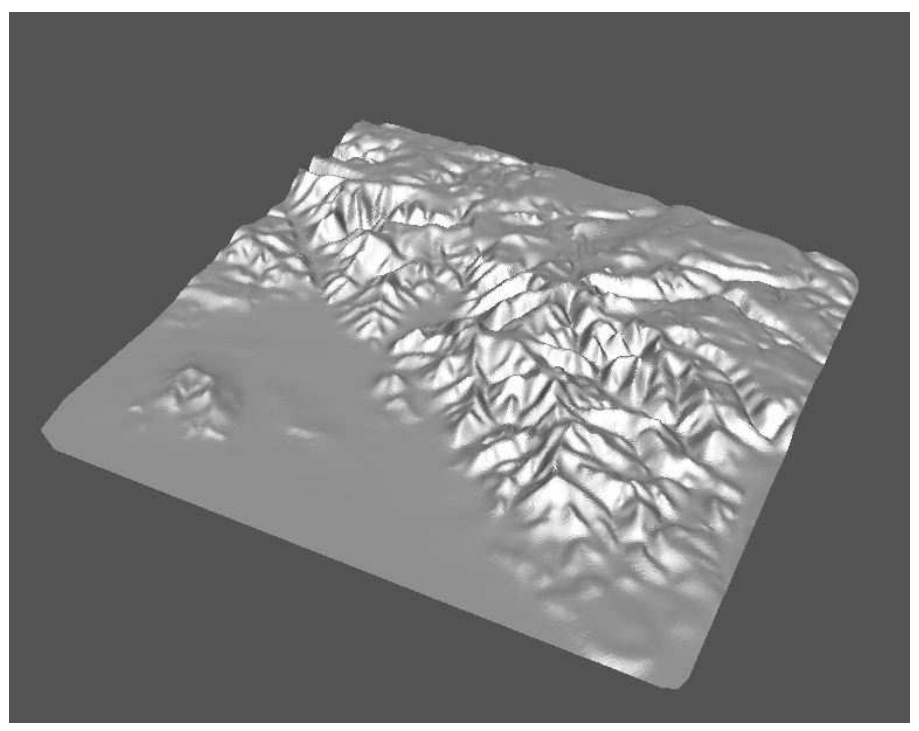

Fig. 6. The minimal energy interpolant of the Black Forest data.

- Time: The main use of the DDC algorithm is to make it possible to solve large variational spline problems which could not be solved at all without using the method. For small problems, it can take more time to solve for a DDC leastsquares spline than for the global least-squares spline itself. However, even for this moderately sized problem, we see that most of the DDC splines took less time to compute for nearly the same accuracy. 


\begin{tabular}{||c||c||c||c||c||c||c||}
\hline$m$ & $k$ & $e_{\infty}$ & $e_{2}$ & $e_{\infty}^{c}$ & $e_{2}^{c}$ & time \\
\hline 1 & 0 & $4.5(-7)$ & $2.3(-8)$ & & & 42 \\
4 & 1 & $4.7(-6)$ & $7.1(-8)$ & $1.9(-5)$ & $2.1(-8)$ & 44 \\
4 & 2 & $3.8(-6)$ & $5.3(-8)$ & $5.6(-6)$ & $1.0(-8)$ & 62 \\
4 & 3 & $9.9(-7)$ & $3.2(-8)$ & $1.7(-6)$ & $5.5(-9)$ & 82 \\
8 & 1 & $5.5(-6)$ & $1.1(-7)$ & $2.0(-5)$ & $4.3(-8)$ & 48 \\
8 & 2 & $3.8(-6)$ & $8.0(-8)$ & $1.1(-5)$ & $2.2(-8)$ & 93 \\
8 & 3 & $1.7(-6)$ & $6.8(-8)$ & $3.9(-6)$ & $1.7(-8)$ & 151 \\
10 & 2 & $2.5(-6)$ & $9.8(-8)$ & $5.3(-6)$ & $2.8(-8)$ & 113 \\
\hline
\end{tabular}

Tab. 3. DDC least-squares fits to Franke's function from $\mathcal{S}_{5}^{1,2}\left(\triangle_{B F}\right)$.

- Condition numbers: The condition numbers of the Gram matrix arising in discrete least-squares fitting with splines is dependent on a number of things. The size of $\beta$ (which reflects whether there are skinny triangles in $\triangle$ ) plays a role, but not as large a role as in the minimal energy case (since here we are not working with second derivatives). What seems more critical in the leastsquares case is the distribution of data over the triangles - if there are triangles with barely enough data to insure a nonsingular system, the condition number tends to be high. For this particular example, the condition numbers of the matrices arising in the subproblems lie in the range of $10^{5}$ to $10^{6}$.

- Shape of the surface: We have compared 3D plots of the global least-squares fit of $f$ with the DDC-least-squares fits for the parameters in Tab. 3. For $k=1$ we noticed slight deviations in shape, but for all higher values of $k$ we got excellent shapes.

\section{$\S 4$. Analytical Tools}

In this section we set the stage for the proofs in Sect. 5 of our main results.

\subsection{Bernstein-Bézier techniques}

We make use of the Bernstein-Bézier representation of splines. Given $d$ and $\triangle$, let $\mathcal{D}_{d, \triangle}:=\cup_{T \in \triangle} \mathcal{D}_{d, T}$ be the corresponding set of domain points, where for each $T:=\left\langle v_{1}, v_{2}, v_{3}\right\rangle$,

$$
\mathcal{D}_{d, T}:=\left\{\xi_{i j k}^{T}:=\frac{i v_{1}+j v_{2}+k v_{3}}{d}\right\}_{i+j+k=d} .
$$

Then every spline $s \in \mathcal{S}_{d}^{0}(\triangle)$ is uniquely determined by its set of coefficients

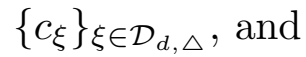

$$
\left.s\right|_{T}:=\sum_{\xi \in \mathcal{D}_{d, T}} c_{\xi} B_{\xi}^{T},
$$


where $\left\{B_{\xi}^{T}\right\}$ are the Bernstein basis polynomials associated with the triangle $T$.

Suppose now that $\mathcal{S}(\triangle)$ is a subspace of $\mathcal{S}_{d}^{0}(\triangle)$. Then a set $\mathcal{M} \subseteq \mathcal{D}_{d, \triangle}$ of domain points is called a minimal determining set (MDS) for $\mathcal{S}(\triangle)$ provided it is the smallest set of domain points such that the corresponding coefficients $\left\{c_{\xi}\right\}_{\xi \in \mathcal{M}}$ can be set independently, and all other coefficients of $s$ can be consistently determined from smoothness conditions, i.e., in such a way that all smoothness conditions are satisfied, see p. 136 of [10]. The dimension of $\mathcal{S}(\triangle)$ is then equal to the cardinality of $\mathcal{M}$. Clearly, $\mathcal{M}=\mathcal{D}_{d, \triangle}$ is a minimal determining set for $\mathcal{S}_{d}^{0}(\triangle)$, and thus the dimension of $\mathcal{S}_{d}^{0}(\triangle)$ is $n_{V}+(d-1) n_{E}+\left(\begin{array}{c}d-1 \\ 2\end{array}\right) n_{T}$, where $n_{V}, n_{E}, n_{T}$ are the number of vertices, edges, and triangles of $\triangle$.

For each $\eta \in \mathcal{D}_{d, \Delta} \backslash \mathcal{M}$, let $\Gamma_{\eta}$ be the smallest subset of $\mathcal{M}$ such that $c_{\eta}$ can be computed from the coefficients $\left\{c_{\xi}\right\}_{\xi \in \Gamma_{\eta}}$ by smoothness conditions. Then $\mathcal{M}$ is called $\ell$-local provided there exists an integer $\ell$ not depending on $\triangle$ such that

$$
\Gamma_{\eta} \subseteq \operatorname{star}^{\ell}\left(T_{\eta}\right), \quad \text { all } \eta \in \mathcal{D}_{d, \Delta} \backslash \mathcal{M},
$$

where $T_{\eta}$ is a triangle containing $\eta . \mathcal{M}$ is said to be stable provided there exists a constant $K_{3}$ depending only on $\ell$ and the smallest angle in the triangulation $\triangle$ such that

$$
\left|c_{\eta}\right| \leq K_{3} \max _{\xi \in \Gamma_{\eta}}\left|c_{\xi}\right|, \quad \text { all } \eta \in \mathcal{D}_{d, \triangle} \backslash \mathcal{M} .
$$

Suppose $\mathcal{M}$ is a stable local MDS for $\mathcal{S}(\triangle)$. For each $\xi \in \mathcal{M}$, let $\psi_{\xi}$ be the spline in $\mathcal{S}(\triangle)$ such that $c_{\xi}=1$ while $c_{\eta}=0$ for all other $\eta \in \mathcal{M}$. Then the splines $\left\{\psi_{\xi}\right\}_{\xi \in \mathcal{M}}$ are clearly linearly independent and form a basis for $\mathcal{S}(\triangle)$. This basis is called the $\mathcal{M}$-basis for $\mathcal{S}(\triangle)$, see Sect. 5.8 of [10]. It is stable and $\ell$-local in the sense that for all $\xi \in \mathcal{M}$,

$$
\left\|\psi_{\xi}\right\|_{\Omega} \leq K_{4},
$$

and

$$
\operatorname{supp} \psi_{\xi} \subseteq \operatorname{star}^{\ell}\left(T_{\xi}\right),
$$

where $T_{\xi}$ is a triangle containing $\xi$. Here $\ell$ is the integer constant in (4.1), and the constant $K_{4}$ depends only on $\ell$ and the smallest angle in $\triangle$.

There are many spaces with stable local bases. For example, the spaces $\mathcal{S}_{d}^{0}(\triangle)$ have stable local bases with $\ell=1$. The same is true for the superspline spaces $\mathcal{S}_{4 r+1}^{r, 2 r}(\triangle)$ for all $r \geq 1$. There are also several families of macro-element spaces defined for all $r \geq 1$ with the same property, see [10].

\subsection{Two lemmas}

For convenience we recall a lemma from [3].

Lemma 4.1. Suppose $a_{0}, a_{1}, \cdots$, are nonnegative numbers such that

$$
\gamma \sum_{j \geq \nu} a_{j} \leq a_{\nu}, \quad \text { for all } \nu=0,1,2, \cdots,
$$


for some $0<\gamma<1$. Then $a_{\nu} \leq \frac{1}{\gamma} \sigma^{\nu} a_{0}$, where $\sigma:=1-\gamma$.

We now establish a key lemma whose proof is modelled on the proof of Theorem 3.1 in [6]. Let $\mathcal{W}$ be a space of spline functions defined on a triangulation $\triangle$ of $\Omega$ with inner product $\langle f, g\rangle_{\mathcal{W}}$ and norm $\|f\|_{\mathcal{W}}^{2}:=\langle f, f\rangle_{\mathcal{W}}$. Suppose that $\left\{\mathcal{B}_{\xi}\right\}_{\xi \in \mathcal{M}}$ is a 1-local basis for $\mathcal{W}$ such that for some constants $C_{1}, C_{2}$,

$$
C_{1} \sum_{\xi \in \mathcal{M}}\left|c_{\xi}\right|^{2} \leq\left\|\sum_{\xi \in \mathcal{M}} c_{\xi} B_{\xi}\right\|_{\mathcal{W}}^{2} \leq C_{2} \sum_{\xi \in \mathcal{M}}\left|c_{\xi}\right|^{2}
$$

for all coefficient vectors $c:=\left\{c_{\xi}\right\}_{\xi \in \mathcal{M}}$.

Lemma 4.2. Let $\omega$ be a cluster of triangles in $\triangle$, and let $T \in \omega$. Then there exists constants $0<\sigma<1$ and $C$ depending only on the ratio $C_{2} / C_{1}$ such that if $g$ is a function in $\mathcal{W}$ with

$$
\langle g, w\rangle_{\mathcal{W}}=0, \quad \text { for all } w \in \mathcal{W} \text { with } \operatorname{supp}(w) \subseteq \operatorname{star}^{k}(\omega)
$$

for some $k \geq 1$, then

$$
\left\|g \cdot \chi_{T}\right\|_{\mathcal{W}} \leq C \sigma^{k}\|g\|_{\mathcal{W}}
$$

Proof: For each $\nu \geq 0$, let

$$
\mathcal{M}_{\nu}^{\omega}:=\left\{\xi \in \mathcal{M}: \operatorname{supp}\left(B_{\xi}\right) \subseteq \operatorname{star}^{\nu}\left(\mathbb{R}^{2} \backslash \operatorname{star}^{k}(\omega)\right)\right\}
$$

Define $\mathcal{N}_{0}^{\omega}:=\mathcal{M}_{0}^{\omega}$, and let $\mathcal{N}_{\nu}^{\omega}:=\mathcal{M}_{\nu}^{\omega} \backslash \mathcal{M}_{\nu-1}^{\omega}$, for $\nu \geq 1$. Given $g:=\sum_{\xi \in \mathcal{M}} c_{\xi} B_{\xi}$, let

$$
g_{\nu}:=\sum_{\xi \in \mathcal{M}_{\nu}^{\omega}} c_{\xi} B_{\xi}, \quad u_{\nu}:=g-g_{\nu}, \quad a_{\nu}:=\sum_{\xi \in \mathcal{N}_{\nu}^{\omega}} c_{\xi}^{2} .
$$

By (4.6),

$$
\sum_{j \geq \nu+1} a_{j}=\sum_{\xi \notin \mathcal{M}_{\nu}^{\omega}} c_{\xi}^{2} \leq \frac{\left\|u_{\nu}\right\|_{\mathcal{W}}^{2}}{C_{1}}
$$

while (4.7) implies $\left\langle g, u_{\nu}\right\rangle_{\mathcal{W}}=0$. Since $\operatorname{supp}\left(u_{\nu}\right) \cap \bigcup_{\xi \in \mathcal{M}_{\nu-1}^{\omega}} \operatorname{supp}\left(B_{\xi}\right)=\emptyset$ for $\nu \geq 1$, it follows that

$$
\begin{aligned}
\left\|u_{\nu}\right\|_{\mathcal{W}}^{2} & =\left\langle g-g_{\nu}, u_{\nu}\right\rangle_{\mathcal{W}}=-\left\langle g_{\nu}, u_{\nu}\right\rangle_{\mathcal{W}} \\
& =-\left\langle\sum_{\xi \in \mathcal{N}_{\nu}^{\omega}} c_{\xi} B_{\xi}, u_{\nu}\right\rangle_{\mathcal{W}} \leq\left\|\sum_{\xi \in \mathcal{N}_{\nu}^{\omega}} c_{\xi} B_{\xi}\right\|_{\mathcal{W}}\left\|u_{\nu}\right\|_{\mathcal{W}} .
\end{aligned}
$$

Dividing by $\left\|u_{\nu}\right\|_{\mathcal{W}}$ and squaring, then using (4.6), we get

$$
\left\|u_{\nu}\right\|_{\mathcal{W}}^{2} \leq\left\|\sum_{\xi \in \mathcal{N}_{\nu}^{\omega}} c_{\xi} B_{\xi}\right\|_{\mathcal{W}}^{2} \leq C_{2} a_{\nu}
$$


Combining (4.9) and (4.10) gives

$$
\sum_{j \geq \nu} a_{j} \leq \frac{C_{1}+C_{2}}{C_{1}} a_{\nu}, \quad \nu \geq 1 .
$$

Then applying Lemma 4.1 gives

$$
a_{\nu} \leq \frac{\left(C_{1}+C_{2}\right)}{C_{1}} \sigma^{\nu-1} a_{1},
$$

with $\sigma:=C_{2} /\left(C_{1}+C_{2}\right)$. On the other hand,

$$
a_{1} \leq \sum_{j \geq 0} a_{j}=\sum_{\xi \in \mathcal{M}} c_{\xi}^{2} \leq \frac{1}{C_{1}}\|g\|_{\mathcal{W}}^{2} .
$$

Now let $q$ be the smallest integer such that there is a basis function $B_{\xi}$ in $\mathcal{M}_{q}^{\omega}$ with $T \subseteq \operatorname{supp}\left(B_{\xi}\right)$. Then by $(4.6)$,

$$
\begin{aligned}
\left\|g \cdot \chi_{T}\right\|_{\mathcal{W}}^{2} & =\left\|\sum_{\left.B_{\xi}\right|_{T} \neq 0} c_{\xi} B_{\xi}\right\|_{\mathcal{W}}^{2} \leq C_{2} \sum_{\xi \notin \mathcal{M}_{q-1}^{\omega}} c_{\xi}^{2}=C_{2} \sum_{j \geq q} a_{j} \\
& \leq \frac{C_{2}}{C_{1}}\left(\frac{C_{1}+C_{2}}{C_{1}}\right)^{2} \sigma^{q-1}\|g\|_{\mathcal{W}}^{2} .
\end{aligned}
$$

Since $q \geq k+1$, we have (4.8).

\section{$\S 5$. Dependence of the Errors on the Parameter $k$}

In this section we examine the difference between global splines and their DDC approximations as a function of the parameter $k$. We give separate results for ME, DLS, and PLS splines. Throughout the section we assume that $\triangle$ is a $\beta$-uniform triangulation, and that $\mathcal{S}(\triangle)$ is an associated spline space with a stable local $\mathcal{M}$ basis.

\subsection{Minimal energy interpolating splines}

Given a set of measurements $\left\{f_{i}\right\}_{i=1}^{n_{d}}$ of a function $f$ at the vertices of a triangulation $\triangle$, let $s_{E}$ be the corresponding minimal energy interpolating spline. Let $s_{E}^{k}$ be the DDC minimal energy spline computed using Algorithm 1.1 with parameter $k$. In (2.8) we showed that if $f \in W_{\infty}^{2}(\Omega)$, then $\left\|s_{E}-s_{E}^{k}\right\|_{\Omega}=\mathcal{O}\left(|\triangle|^{2}\right)$. In this section we discuss the dependence of this difference on $k$.

Theorem 5.1. There exists $\sigma \in(0,1)$ such that for all $f \in W_{\infty}^{2}(\Omega)$,

$$
\left\|D_{x}^{\alpha} D_{y}^{\beta}\left(s_{E}-s_{E}^{k}\right)\right\|_{\Omega} \leq C \sigma^{k}|\triangle|^{1-\alpha-\beta}|f|_{2, \Omega},
$$


for all $0 \leq \alpha+\beta \leq 1$. When $\Omega$ is convex, $C$ is a constant depending only on $d, \ell, \beta, \theta_{\triangle}$ and the area of $\Omega$. When $\Omega$ is nonconvex, $C$ also depends on the Lipschitz constant of the boundary of $\Omega$.

Proof: Let $\Omega_{i}$ be one of the subdomains in Algorithm 1.1. In view of the way in which $s_{E}$ is defined, it suffices to estimate $\left\|s_{E}-s_{E}^{k}\right\|_{\Omega_{i}}$. Let $\triangle_{i}^{k}$ be the subtriangulation obtained by restricting $\triangle$ to $\Omega_{i}^{k}:=\operatorname{star}^{k}\left(\Omega_{i}\right)$. Fix $k \geq 1$. We make use of Lemma 4.2 applied to

$$
\mathcal{W}=\left\{\left.s \in \mathcal{S}(\triangle)\right|_{\Omega_{i}^{k}}: s(v)=0, \text { all vertices } v \text { of } \triangle_{i}^{k}\right\},
$$

with the inner product

$$
\langle\phi, \psi\rangle_{E, \Omega_{i}^{k}}:=\int_{\Omega_{i}^{k}}\left[\phi_{x x} \psi_{x x}+2 \phi_{x y} \psi_{x y}+\phi_{y y} \psi_{y y}\right] d x d y .
$$

Let $s_{E, \Omega_{i}^{k}}:=\left.s_{E}\right|_{\Omega_{i}^{k}}$ be the global minimal energy interpolant of $f$ restricted to $\Omega_{i}^{k}$, and let $s_{E, i}^{k}$ be the minimal energy interpolant of $f$ in the space $\left.\mathcal{S}(\triangle)\right|_{\Omega_{i}^{k}}$. Let $\left\{B_{\xi}\right\}_{\xi \in \mathcal{M}_{i k}}$ be a stable 1-local basis for $\left.\mathcal{S}(\triangle)\right|_{\Omega_{i}^{k}}$. It was shown in Corollary 5.3 of [7] that

$$
C_{1}|\triangle|^{-2} \sum_{\xi \in \mathcal{M}_{i}^{k}}\left|c_{\xi}\right|^{2} \leq\left\|\sum_{\xi \in \mathcal{M}_{i}^{k}} c_{\xi} B_{\xi}\right\|_{E, \Omega_{i}^{k}} \leq C_{2}|\triangle|^{-2} \sum_{\xi \in \mathcal{M}_{i}^{k}}\left|c_{\xi}\right|^{2},
$$

where $C_{1}$ and $C_{2}$ depend only on $d, \ell$ and $\beta$. Writing $g:=s_{E, \Omega_{i}^{k}}-s_{E, i}^{k} \in \mathcal{W}$, and using the characterization of minimal energy splines, we have

$$
\left\langle g, B_{\xi}\right\rangle_{E, \Omega_{i}^{k}}=0, \quad \text { all } B_{\xi} \text { with } \operatorname{supp}\left(B_{\xi}\right) \subseteq \Omega_{i}^{k} .
$$

Now suppose $T$ is a triangle in $\Omega_{i}$ where $|g|$ takes its maximum. Since $g$ is a polynomial on $T$, we can use Lemma 6.1 of [7] and Theorem 1.1 of [10] to get

$$
\|g\|_{\Omega_{i}}=\|g\|_{T} \leq 12|T|^{2}|g|_{2, \infty, T} \leq C_{3}|\triangle||g|_{2,2, T} \leq C_{3}|\triangle|\left\|g \cdot \chi_{T}\right\|_{E, \Omega_{i}^{k}},
$$

where $C_{3}$ depends only on $d$. In view of (5.3) and (5.4), we can apply Lemma 4.2 to get

$$
\left\|g \cdot \chi_{T}\right\|_{E, \Omega_{i}^{k}} \leq C_{4} \sigma^{k}\|g\|_{E, \Omega_{i}^{k}} \leq C_{4} A^{1 / 2} \sigma^{k}|g|_{2, \infty, \Omega_{i}^{k}},
$$

where $A$ is the area of $\Omega_{i}^{k}$. Note that $C_{4}$ does not depend on $|\triangle|$ since the constant in Lemma 4.2 depends on the ratio $C_{2}|\triangle|^{-2} / C_{1}|\triangle|^{-2}$. Now let $\tau$ be a triangle where $|g|_{2, \infty, \Omega_{i}}$ takes its maximum. Then using the Markov inequality, we have

$$
|g|_{2, \infty, \Omega_{i}}=|g|_{2, \infty, \tau} \leq \frac{C_{5}}{|\tau|^{2}}\|g\|_{\tau} \leq \frac{C_{5}}{|\tau|^{2}}\left(\left\|f-s_{E}\right\|_{\tau}+\left\|f-s_{E, i}^{k}\right\|_{\tau}\right) .
$$

Combining the inequalities (5.5)-(5.7) with the error bound (2.7), we get (5.1) for $\alpha=\beta=0$. To get the result for derivatives, we apply the Markov inequality on a triangle where $\left\|D_{x}^{\alpha} D_{y}^{\beta} g\right\|_{\Omega}$ takes its maximum value. 


\subsection{Discrete least-squares splines}

Given a set of measurements $\left\{f_{i}\right\}_{i=1}^{n_{d}}$ of a function $f$ and a triangulation $\triangle$, let $s_{L}$ be the discrete least-squares spline fit of $f$ from $\mathcal{S}(\triangle)$. Let $s_{L}^{k}$ be the DDC leastsquares spline produced by Algorithm 1.1 with parameter $k$. In (2.14) we showed that if $f \in W_{\infty}^{m+1}(\Omega)$, then $\left\|s_{L}-s_{L}^{k}\right\|_{\Omega}=\mathcal{O}\left(|\triangle|^{m+1}\right)$. In this section we discuss the dependence of this difference on $k$. The following result gives results for the derivatives of the difference. As is customary in spline theory, the norm here is to be interpreted as the maximum of the supremum norms over the triangles in $\triangle$ since the splines $s_{L}$ and $s_{L}^{k}$ may not have derivatives at every point in $\Omega$.

Theorem 5.2. There exists $\sigma \in(0,1)$ such that if $f \in W_{\infty}^{m+1}(\Omega)$ with $0 \leq m \leq d$, then

$$
\left\|D_{x}^{\alpha} D_{y}^{\beta}\left(s_{L}-s_{L}^{k}\right)\right\|_{\Omega} \leq C \sigma^{k}|\triangle|^{m-\alpha-\beta}|f|_{m+1, \Omega} .
$$

for all $0 \leq \alpha+\beta \leq m$. When $\Omega$ is convex, $C$ is a constant depending only on $d, \ell, \beta, K_{1}, K_{2}$ and $\theta_{\triangle}$. When $\Omega$ is nonconvex, $C$ also depends on the Lipschitz constant of the boundary of $\Omega$.

Proof: Let $\Omega_{i}$ be one of the subdomains in Algorithm 1.1. In view of the way in which $s_{L}$ is defined, it suffices to estimate the norm of $s_{L}-s_{L}^{k}$ on $\Omega_{i}$. Let $\triangle_{i}^{k}$ be the subtriangulation obtained by restricting $\triangle$ to $\Omega_{i}^{k}:=\operatorname{star}^{k}\left(\Omega_{i}\right)$. Fix $k \geq 1$. We make use of Lemma 4.2 applied to $\mathcal{W}=\left.\mathcal{S}(\triangle)\right|_{\Omega_{i}^{k}}$ with the inner product

$$
\langle\phi, \psi\rangle_{\mathcal{A}_{i}^{k}}:=\sum_{\left(x_{i}, y_{i}\right) \in \Omega_{i}^{k}} \phi\left(x_{i}, y_{i}\right) \psi\left(x_{i}, y_{i}\right) .
$$

Let $s_{L, \Omega_{i}^{k}}:=\left.s_{L}\right|_{\Omega_{i}^{k}}$ be the restriction to $\Omega_{i}^{k}$ of the global least-squares spline fit $s_{L}$ of $f$ from $\mathcal{S}(\triangle)$, and let $s_{L, i}^{k}$ be the least-squares spline fit of $f$ from the space $\left.\mathcal{S}(\triangle)\right|_{\Omega_{i}^{k}}$. Let $\left\{B_{\xi}\right\}_{\xi \in \mathcal{M}_{i k}}$ be a stable 1-local basis for $\left.\mathcal{S}(\triangle)\right|_{\Omega_{i}^{k}}$. It was shown in Lemma 5.1 of $[6]$ that

$$
C_{1} \sum_{\xi \in \mathcal{M}_{i}^{k}}\left|c_{\xi}\right|^{2} \leq\left\|\sum_{\xi \in \mathcal{M}_{i}^{k}} c_{\xi} B_{\xi}\right\|_{\mathcal{A}_{i}^{k}} \leq C_{2} \sum_{\xi \in \mathcal{M}_{i}^{k}}\left|c_{\xi}\right|^{2},
$$

Writing $g:=s_{L, \Omega_{i}^{k}}-s_{L, i}^{k} \in \mathcal{W}$, and using the characterization of least-squares splines, we have

$$
\left\langle g, B_{\xi}\right\rangle_{\mathcal{A}_{i}^{k}}=0, \quad \text { all } B_{\xi} \text { with } \operatorname{supp}\left(B_{\xi}\right) \subseteq \Omega_{i}^{k} .
$$

Now suppose $T$ is a triangle in $\Omega_{i}$ where $|g|$ takes its maximum. Then using (2.12) and Lemma 4.2 we get

$$
\|g\|_{\Omega_{i}}=\|g\|_{T} \leq \frac{1}{K_{1}}\left\|g \cdot \chi_{T}\right\|_{\mathcal{A}_{i}^{k}} \leq \frac{C_{3}}{K_{1}} \sigma^{k}\|g\|_{\mathcal{A}_{i}^{k}} \leq \frac{C_{3} \sqrt{N K_{2}}}{K_{1}} \sigma^{k}\|g\|_{\Omega_{i}^{k}},
$$


where $N$ is the number of triangles in $\Omega_{i}^{k}$. Note that $\sqrt{N} \leq C_{4} /|\triangle|$, where $C_{4}$ depends on the area of $\Omega_{i}^{k}$ and the constant $\beta$. On the other hand,

$$
\|g\|_{\Omega_{i}^{k}} \leq\left\|f-s_{L}\right\|_{\Omega_{i}^{k}}+\left\|f-s_{L, i}^{k}\right\|_{\Omega_{i}^{k}} .
$$

Combining the last two inequalities with the error bound (2.13), we get (5.8) for $\alpha=\beta=0$. To get the result for the derivative $D_{x}^{\alpha} D_{y}^{\beta}$, we apply the Markov inequality to a triangle where $\left\|D_{x}^{\alpha} D_{y}^{\beta} g\right\|_{\Omega}$ takes its maximum.

\subsection{Penalized least-squares splines}

Given a set of measurements $\left\{f_{i}\right\}_{i=1}^{n_{d}}$ of a function $f$ and a triangulation $\triangle$, let $s_{\lambda}$ be the penalized least-squares spline fit of $f$ from $\mathcal{S}(\triangle)$ with smoothing parameter $\lambda>0$. Let $s_{\lambda}^{k}$ be the DDC penalized least-squares spline produced by Algorithm 1.1 with parameter $k$. In $(2.17)$ we showed that if $f \in W_{\infty}^{m+1}(\Omega)$, then $\left\|s_{\lambda}-s_{\lambda}^{k}\right\|_{\Omega}=$ $\mathcal{O}\left(\mid \triangle^{m+1}\right)+\mathcal{O}(\lambda)$. In this section we discuss the dependence of this difference on $k$.

Theorem 5.3. There exists $\sigma \in(0,1)$ such that if $f \in W_{\infty}^{m+1}(\Omega)$ with $1 \leq m \leq d$, then

$$
\left\|s_{\lambda}-s_{\lambda}^{k}\right\|_{\Omega} \leq C \sigma^{k}\left(1+\frac{\sqrt{\lambda}}{|\triangle|}\right)\left(|\triangle|^{m}|f|_{m+1, \Omega}+\frac{\lambda}{|\triangle|}|f|_{2, \Omega}\right) .
$$

if $\lambda$ is sufficiently small compared to $|\triangle|$. When $\Omega$ is convex, $C$ is a constant depending only on $d, \ell, \beta, K_{1}, K_{2}, \theta_{\triangle}$ and the area of $\Omega$. When $\Omega$ is nonconvex, $C$ also depends on the Lipschitz constant of the boundary of $\Omega$.

Proof: Let $\Omega_{i}$ be one of the subdomains in Algorithm 1.1. In view of the way in which $s_{\lambda}$ is defined, it suffices to estimate the norm of $s_{\lambda}-s_{\lambda}^{k}$ on $\Omega_{i}$. Let $\triangle_{i}^{k}$ be the subtriangulation obtained by restricting $\triangle$ to $\Omega_{i}^{k}:=\operatorname{star}^{k}\left(\Omega_{i}\right)$. Fix $k \geq 1$. We make use of Lemma 4.2 applied to $\mathcal{W}:=\left.\mathcal{S}(\triangle)\right|_{\Omega_{i}^{k}}$ with the inner product

$$
\langle\phi, \psi\rangle_{\lambda}:=\langle\phi, \psi\rangle_{\mathcal{A}_{i}^{k}}+\lambda\langle\phi, \psi\rangle_{E, \Omega_{i}^{k}},
$$

where the inner-products in this definition are as in (5.2) and (5.9). Let $s_{\lambda, \Omega_{i}^{k}}:=$ $\left.s_{\lambda}\right|_{\Omega_{i}^{k}}$ be the restriction to $\Omega_{i}^{k}$ of the global penalized least-squares spline fit $s_{\lambda}$ of $f$ from $\mathcal{S}(\triangle)$, and let $s_{\lambda, i}^{k}$ be the penalized least-squares spline fit of $f$ from the space $\left.\mathcal{S}(\triangle)\right|_{\Omega_{i}^{k}}$ using data in $\Omega_{i}^{k}$. Let $\left\{B_{\xi}\right\}_{\xi \in \mathcal{M}_{i k}}$ be a stable 1-local basis for $\left.\mathcal{S}(\triangle)\right|_{\Omega_{i}^{k}}$ as in the proof of Theorem 5.2. Combining (5.3) and (5.10), we see that

$$
C_{1}\left(1+\frac{\lambda}{|\triangle|^{2}}\right) \sum_{\xi \in \mathcal{M}_{i}^{k}}\left|c_{\xi}\right|^{2} \leq\left\|\sum_{\xi \in \mathcal{M}_{i}^{k}} c_{\xi} B_{\xi}\right\|_{\lambda} \leq C_{2}\left(1+\frac{\lambda}{|\triangle|^{2}}\right) \sum_{\xi \in \mathcal{M}_{i}^{k}}\left|c_{\xi}\right|^{2} .
$$

Writing $g:=s_{\lambda, \Omega_{i}^{k}}-s_{\lambda, i}^{k} \in \mathcal{W}$, and using the characterization of penalized leastsquares splines, we have

$$
\left\langle g, B_{\xi}\right\rangle_{\lambda}=0, \quad \text { all } B_{\xi} \text { with } \operatorname{supp}\left(B_{\xi}\right) \subseteq \Omega_{i}^{k} .
$$


Now suppose $T$ is a triangle in $\Omega_{i}$ where $|g|$ takes its maximum. Then by $(2.12)$,

$$
\|g\|_{T} \leq \frac{1}{K_{1}}\left\|g \cdot \chi_{T}\right\|_{\mathcal{A}_{i}^{k}} \leq \frac{1}{K_{1}}\left(\left\|g \cdot \chi_{T}\right\|_{\mathcal{A}_{i}^{k}}^{2}+\lambda\left\|g \cdot \chi_{T}\right\|_{E, \Omega_{i}^{k}}^{2}\right)^{1 / 2}=\frac{1}{K_{1}}\left\|g \cdot \chi_{T}\right\|_{\lambda} .
$$

Using Lemma 4.2, we get

$\|g\|_{T} \leq \frac{C_{3}}{K_{1}} \sigma^{k}\|g\|_{\lambda} \leq \frac{C_{3}}{K_{1}} \sigma^{k}\left(\|g\|_{\mathcal{A}_{i}^{k}}^{2}+\lambda\|g\|_{E, \Omega_{i}^{k}}^{2}\right)^{1 / 2} \leq \frac{C_{3}}{K_{1}} \sigma^{k}\left(\|g\|_{\mathcal{A}_{i}^{k}}+\sqrt{\lambda}\|g\|_{E, \Omega_{i}^{k}}\right)$

where $C_{3}$ depends only on the ratio $C_{2} / C_{1}$. Following the proofs of Theorems 5.1 and 5.2 , we see that

$$
\|g\|_{E, \Omega_{i}^{k}} \leq \frac{C_{4}}{|\triangle|^{2}}\|g\|_{\Omega_{i}^{k}}, \quad\|g\|_{A_{i}^{k}} \leq \frac{C_{5}}{|\triangle|}\|g\|_{\Omega_{i}^{k}}
$$

which gives

$$
\|g\|_{T} \leq C_{6} \sigma^{k}\left(\frac{1}{|\triangle|}+\frac{\sqrt{\lambda}}{|\triangle|^{2}}\right)\|g\|_{\Omega_{i}^{k}} .
$$

Now

$$
\|g\|_{\Omega_{i}^{k}} \leq\left\|f-s_{\lambda}\right\|_{\Omega_{i}^{k}}+\left\|f-s_{\lambda, i}^{k}\right\|_{\Omega_{i}^{k}}
$$

and using (2.16) we get (5.14).

\section{§. Remarks}

Remark 1. Domain decomposition methods have been studied for more than 150 years in the literature on the numerical solution of boundary value problems, going back at least to Schwarz's alternating method, see e.g. [11]. For a comprehensive treatment and an extensive list of references, see [13]. The idea of domain decomposition has recently been adapted to the problem of fitting scattered data with radial basis functions, see [2], as well as to meshless methods (based on radial basis functions) for solving boundary-value problems, see [4] and the book [5].

Remark 2. Many authors have tried to solve global fitting problems by dividing the domain into subdomains, computing fits on each subdomain, and then blending the resulting surface patches together with some kind of blending functions. In most of these methods the use of blending functions changes the form of the final approximant and produces a fit which may not be close to the global fit. Our domain decomposition method is not based on blending functions, and our theorems insure that the DDC-spline is close to the global fit.

Remark 3. As observed in [12], in computation with $\mathcal{M}$-bases it is important to exercise some care in choosing the minimal determining set $\mathcal{M}$. Thus, for example in Fig. 1, for each vertex $v$, the six black dots should be chosen in the triangle with largest angle at $v$. This means that the minimal determining sets for the subspaces $\mathcal{S}\left(\triangle_{i}^{k}\right)$ may not be subsets of the minimal determining set for the full space. 
Remark 4. For convenience, the results of Sect. 5 assume that we are working with a spline space with a 1-local stable basis. However, the same analysis can be carried out with spline spaces with $\ell$-local stable bases under the assumption that $k \geq \ell$.

Remark 5. The computations reported here were done on a Macintosh G5 computer using Fortran. The codes have not been optimized for storage or computational speed. We report computational times to give a feeling for how quickly domain-decomposition spline fits can be computed, and to provide a basis for comparing various algorithms. Since the local fits in the DDC method can be computed independently, the actual run times can be greatly reduced by working on a multiprocessor machine (or on a cluster).

\section{References}

1. Awanou, G., M.-J. Lai, and P. Wenston, The multivariate spline method for scattered data fitting and numerical solution of partial differential equations, in Wavelets and Splines: Athens 2005, G. Chen and M.-J. Lai (eds), Nashboro Press, Brentwood, 2006, 24-74.

2. Beatson, R. K., W. A. Light, and S. Billings, Fast solution of the radial basis function interpolation equations: domain decomposition methods, SIAM J. Sci. Comput. 22 (2000), 1717-1740.

3. de Boor, C., A bound on the $L_{\infty}$-norm of $L_{2}$ approximation by splines in terms of a global mesh ratio, Math. Comp. 30 (1976), 765-711.

4. Duan, Y., Meshless Galerkin method using radial basis functions based on domain decomposition, Appl. Math. Comput. 179 (2006), 750-762.

5. Fasshauer, G., Meshfree Approximation Methods with MATLAB, World Scientific, Singapore, 2007.

6. Golitschek, M. and L. L. Schumaker, Bounds on projections onto bivariate polynomial spline spaces with stable bases, Constr. Approx. 18 (2002), 241254.

7. Golitschek, M. von, M.-J. Lai, and L. L. Schumaker, Error bounds for minimal energy bivariate polynomial splines, Numer. Math. 93 (2002), 315-331.

8. Lai, M.-J., Multivariate splines for data fitting and approximation, in Approximation Theory XII: San Antonio 2007, M. Neamtu and L. L. Schumaker (eds.), Nashboro Press (Brentwood), 2008, 210-228.

9. Lai, M.-J. and L. L. Schumaker, On the approximation power of bivariate splines, Advances in Comp. Math. 9 (1998), 251-279.

10. Lai, M.-J. and L. L. Schumaker, Spline Functions on Triangulations, Cambridge University Press, Cambridge, 2007.

11. Lai, M.-J. and P. Wenston, On Schwarz's domain decomposition methods for elliptic boundary value problems, Numerische Mathematik 84 (2000), 475-495. 
12. Schumaker, L. L., Computing bivariate splines in scattered data fitting and the finite-element method, Numer. Algs. 48 (2008), 237-260.

13. Torseli, A. and O. Widlund, Domain Decomposition Methods - Algorithms and Theory, Springer-Verlag, Berlin, 2005. 